\title{
Upregulation of hsa_circRNA_102958 Indicates Poor Prognosis and Promotes Ovarian Cancer Progression Through miR-1205/SH2D3A Axis
}

This article was published in the following Dove Press journal: Cancer Management and Research

\author{
Guan Wang ${ }^{l, *}$ \\ Huijing Zhang ${ }^{1, *}$ \\ Peiling $\mathrm{Li}^{2}$ \\ 'Department of Gynecology, Heilongjiang \\ Provincial Hospital, Harbin 150000 , \\ People's Republic of China; ${ }^{2}$ Department \\ of Gynecology, The Second Affiliated \\ Hospital of Harbin Medical University, \\ Harbin 150000, People's Republic of \\ China
}

*These authors contributed equally to this work
Correspondence: Peiling Li

Department of Gynecology, The Second Affiliated Hospital of Harbin Medical University, Harbin 150000, People's Republic of China

Email peiling_li@sohu.com
Background: Circular RNA (circRNA) is a recently identified member of noncoding RNAs. It has been demonstrated to regulate gene expression post-transcriptionally and play critical roles in tumorigenesis. However, how circRNA regulates ovarian cancer (OC) progression is poorly understood. Previously, hsa_circRNA_102958 was reported to regulate gastric cancer and colorectal cancer development. This study aims to investigate the role of hsa_circRNA_102958 in OC progression.

Materials and Methods: qRT-PCR was used to test gene expression. CCK8 and colony formation assays were used to analyze proliferation. Transwell assay was utilized to determine migration and invasion. Luciferase reporter assay was conducted to test the interaction between hsa_circRNA_102958 and miR-1205.

Results: hsa_circRNA_102958 was upregulated in OC tissues and cell lines. hsa_circRNA_102958 upregulation indicated a poor prognosis in OC patients. Knockdown of hsa_circRNA_102958 significantly suppressed the proliferation, migration and invasion of OC cells and vice versa. hsa_circRNA_102958 was a competing endogenous RNA (ceRNA) for miR-1205. hsa_circRNA_102958 inhibited miR-1205 activity to promote SH2D3A expression. Overexpression of SH2D3A promoted proliferation, migration and invasion of OC cells.

Conclusion: Our data suggest that hsa_circRNA_102958 promotes OC aggravation through regulation of miR-1205/SH2D3A signaling.

Keywords: ovarian cancer, circular RNA, hsa_circRNA_102958, miR-1205, SH2D3A

\section{Introduction}

Ovarian cancer (OC) is one of the most common malignancies in females and causes a large number of deaths. ${ }^{1}$ The five-year survival rate among OC patients is less than $40 \%$ around the world. ${ }^{2}$ Traditional methods for OC treatment are surgery, chemotherapy and radiotherapy. ${ }^{3}$ Nevertheless, these strategies are difficult to cure this malignancy as most patients were diagnosed at advanced stages with high rate of recurrence and metastasis. ${ }^{4}$ Therefore, it is urgent to identify novel therapeutic targets for OC patients.

Circular RNA (circRNA) is a novel type of noncoding RNA and characterized by a covalently closed continuous loop structure. ${ }^{5}$ The functions of circRNAs have been gradually discovered in cancer. Many studies have showed that circRNAs could be competing endogenous RNAs (ceRNAs) for microRNAs (miRNAs) to regulate gene expression and tumorigenesis. ${ }^{6}$ For example, circABCC2 promotes 
hepatocellular cancer development via sponging miR665 . $^{7}$ Circ_0001730 increases growth and invasiveness of glioma cells through decoying miR-326 to promote Wnt7B expression. ${ }^{8}$ CircHECTD1 promotes gastric cancer development via targeting miR-1256 and activation of $\beta$ catenin/MYC pathway. ${ }^{9}$ Increasing evidences show that quite a number of circRNAs are aberrantly expressed in tumor tissues, including OC. However, the functions of most of them remain undetermined. Thus, it is important to investigate how circRNA regulates carcinogenesis.

A previous study indicates that hsa_circRNA_102958 may be a diagnostic biomarker in gastric cancer. ${ }^{10}$ Recently, there is a research that demonstrates hsa_circRNA_102958 as an oncogene in colorectal cancer. ${ }^{11}$ In the present work, we determined the expression patterns of hsa_circRNA_102958 in OC tissues. We provided evidences to demonstrate that hsa_circRNA_102958 promotes OC cell proliferation, migration and invasion via targeting miR-1205/SH2D3A axis, which may contributes to the development of novel therapeutic targets for $\mathrm{OC}$ treatment.

\section{Materials and Methods}

\section{Human Samples}

$41 \mathrm{OC}$ tissues and their adjacent normal tissues were obtained from the Second Affiliated Hospital of Harbin Medical University. All samples were stored in the liquid nitrogen. Patients received no radiotherapy or chemotherapy before surgery. This work was approved by the Ethics Committee of the Second Affiliated Hospital of Harbin Medical University. Patients provided the written informed consents.

\section{Cell Culture and Transfection}

OC cell lines and IOSE80 cells were purchased from ATCC. Cells were maintained in RPMI-1640 (GIBCO) supplemented with $10 \% \mathrm{FBS}$, penicillin $(100 \mathrm{IU} / \mathrm{mL})$ and streptomycin $(100 \mu \mathrm{g} / \mathrm{mL})$. Small-interfering RNAs targeting hsa_circRNA_102958 or SH2D3A, overexpressing plasmids of hsa_circRNA_102958 or SH2D3A, and miR1205 mimics/inhibitors were designed and purchased from GenePharma. Cells were transfected with above plasmids using Lipofectamine 2000 (Invitrogen, Carlsbad, CA, USA) according to the manufacturer's protocols and used for experiments after $48 \mathrm{~h}$. For rescue assays, hsa_circRNA_102958 siRNA, miR-1205 inhibitors and SH2D3A siRNA were co-transfected into SKOV3 or OVCAR3 cells.

\section{Cell Proliferation Assays}

For CCK- 8 assay, cells $\left(2 \times 10^{3}\right.$ cells per well) were seeded into the 96-well plates. Then cells were cultured for 0,24 , 48 and $72 \mathrm{~h}$. Then $15 \mu \mathrm{L}$ of CCK-8 reagent was added into each well and incubated for $2 \mathrm{~h}$. Finally, the absorbance was determined at $450 \mathrm{~nm}$.

For colony formation assay, 500 cells per well were seeded into the 6-well plates and cultured for 14 days. Then the colony was fixed with $20 \%$ methanol for $30 \mathrm{~min}$ at $25^{\circ} \mathrm{C}$ and stained with $0.5 \%$ crystal violet (Santa Cruz, Dallas, TX, USA) for $15 \mathrm{~min}$ at $25^{\circ} \mathrm{C}$. The colony numbers were finally counted.

\section{qRT-PCR}

TRIzol reagent (Invitrogen, Carlsbad, CA, USA) was used for RNA isolation from OC tissues and cell lines. Then RNAs were utilized for reverse transcription into cDNA. And cDNA was used for qPCR analysis through the SYBR Premix Ex Taq II kit (TaKaRa, Shiga, Japan). U6 or GAPDH was used for normalization. Relative expression was calculated using the $2^{-\Delta \Delta \mathrm{Ct}}$ method (GenePharma, Suzhou, China). Primer sequences were as follows: hsa_circRNA_102958 (Forward, 5'-CACCTCCCTGCAGACAAGAA-3' and reverse, 5'CGTATCCATTCATGCTGGGC-3'), miR-1205 (Forward, 5'CACGCATCTGCAGGGTTT- ${ }^{\prime}$ and reverse, 5'-CCAGTG CAGGGTCCGAGGTA-3'), U6 (Forward, 5'-GCGCGTCGT GAAGCGTTC-3' and reverse, 5'-GTGCAGGGTCCGAGGT -3'), SH2D3A (Forward, 5'-TGAGGGGACTGGTAGAGC TG-3' and reverse, 5'-TTCAGCTCCTGCTCAAAGGC-3') and GAPDH (Forward, 5'-ATGTTGCAACCGGGAAGGA A-3', reverse 5'-AGGAAAAGCATCACCCGGAG-3').

\section{Transwell Assay}

24-well transwell chambers were used for migration and invasion (in the presence of Matrigel). The upper chamber was filled with $200 \mu \mathrm{L}$ serum-free medium and the lower chamber was filled with complete medium. After cultured for $48 \mathrm{~h}$, the cells in the lower chamber was fixed and stained. Cell numbers were then counted within three random fields.

\section{Luciferase Reporter Assay}

The binding between hsa_circRNA_102958 and miR-1205 or between miR-1205 and SH2D3A was predicted using bioinformatics strategy through two online tools (CircInteractome and TargetScan7). Cells were cotransfected with luciferase reporter vector (pMIR-Reporter-hsa_circRNA_102958-WT 
/Mut or pMIR-Reporter-SH2D3A 3'-UTR-WT/Mut) and miR-1205 mimics or negative control (miR-NC). $48 \mathrm{~h}$ later, the luciferase activities were measured using the Dual-Glo Luciferase Assay System (Promega, Fitchburg, WI, USA). Renilla luciferase activity was the normalized control.

\section{Statistical Analysis}

The results are expressed as mean \pm SD. Statistical analysis was conducted using SPSS 20.0. Differences were determined using $t$-test or one-way ANOVA. The difference was statistically significant at $\mathrm{P}<0.05$.

\section{Results}

\section{hsa_circRNA_102958 Was Upregulated in $\mathrm{OC}$}

The expression of hsa_circRNA_102958 was analyzed by qRPCR. The results showed that hsa_circRNA_102958 level was upregulated in 41 OC tissues compared to their adjacent normal tissues (Figure 1A). Besides, hsa_circRNA_102958 expression was also increased in OC cell lines compared to IOSE80 cells (Figure 1B). Importantly, we found that
hsa_circRNA_102958 high expression was associated with a low survival rate in OC patients (Figure 1C), suggesting hsa_circRNA_102958 may be a prognostic biomarker. Then we analyzed its subcellular localization and found that hsa_circRNA_102958 was mainly distributed in the cytoplasm of OC cells (Figure 1D).

\section{hsa_circRNA_102958 Downregulation Inhibited OC Proliferation, Migration and Invasion}

To illustrate the role of hsa_circRNA_102958, we knocked it down via siRNAs (Figure 2A). CCK8 assay showed that hsa_circRNA_102958 downregulation limited the proliferation rates of SKOV3 and OVCAR3 cells (Figure 2B). Consistently, decreased expression of hsa_circRNA_102958 was correlated with fewer numbers of colonies (Figure 2C). Moreover, through Transwell assay, we found that hsa_circRNA_102958 knockdown significantly impaired the migration and invasion of SKOV3 and OVCAR3 cells (Figure 2D and E).

\section{A}

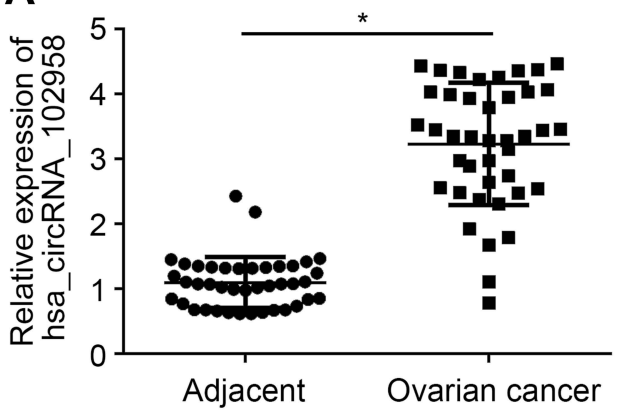

B

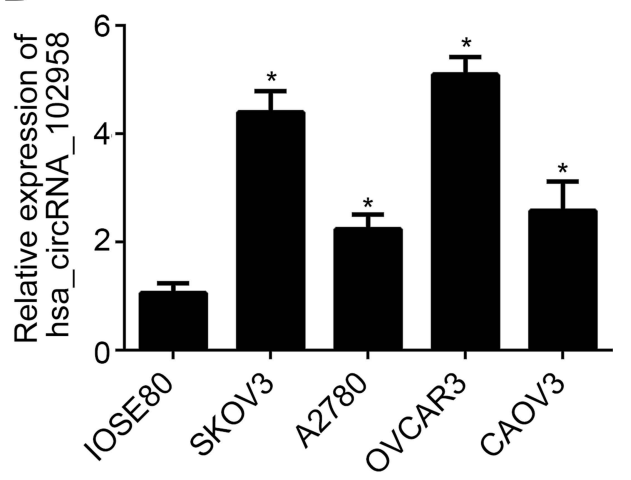

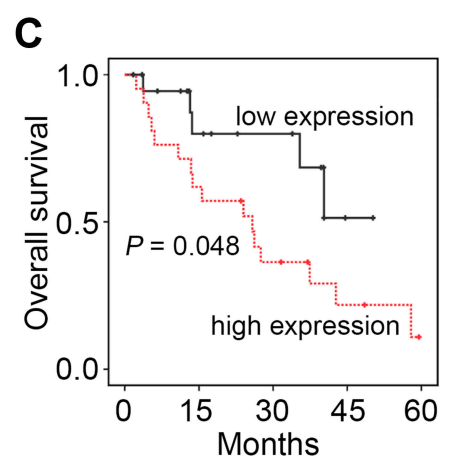

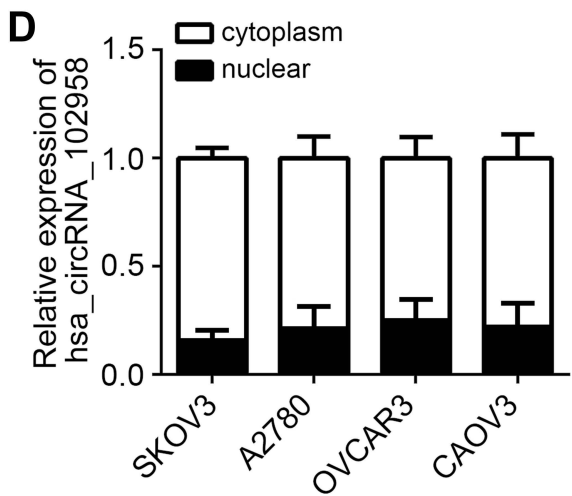

Figure I hsa_circRNA_102958 was upregulated in OC. (A) Relative expression of hsa_circRNA_102958 in pairs of OC tissues and normal controls was analyzed by qPCR. (B) hsa_circRNA_102958 expression in OC cell lines was determined. (C) The overall survival (OS) rate was analyzed in the OC patients based on hsa_circRNA_I02958 expression. (D) Subcellular distribution of hsa_circRNA_102958 was analyzed by qRT-PCR in OC cell lines. $*$ P $<0.05$. 
A

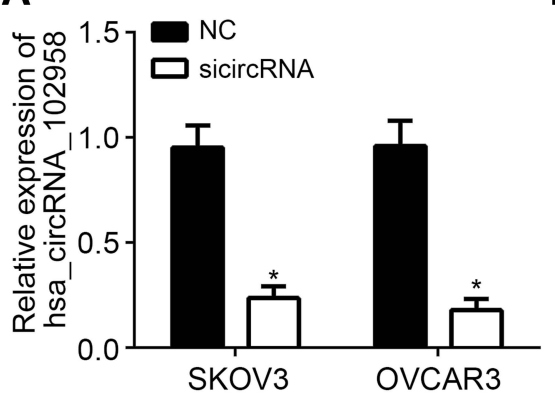

B

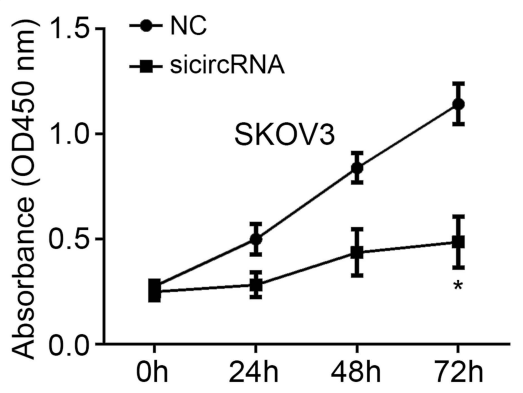

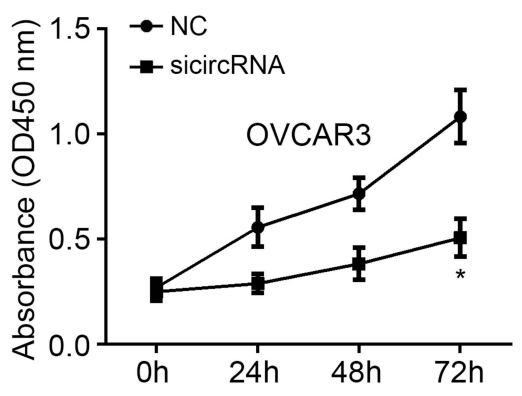
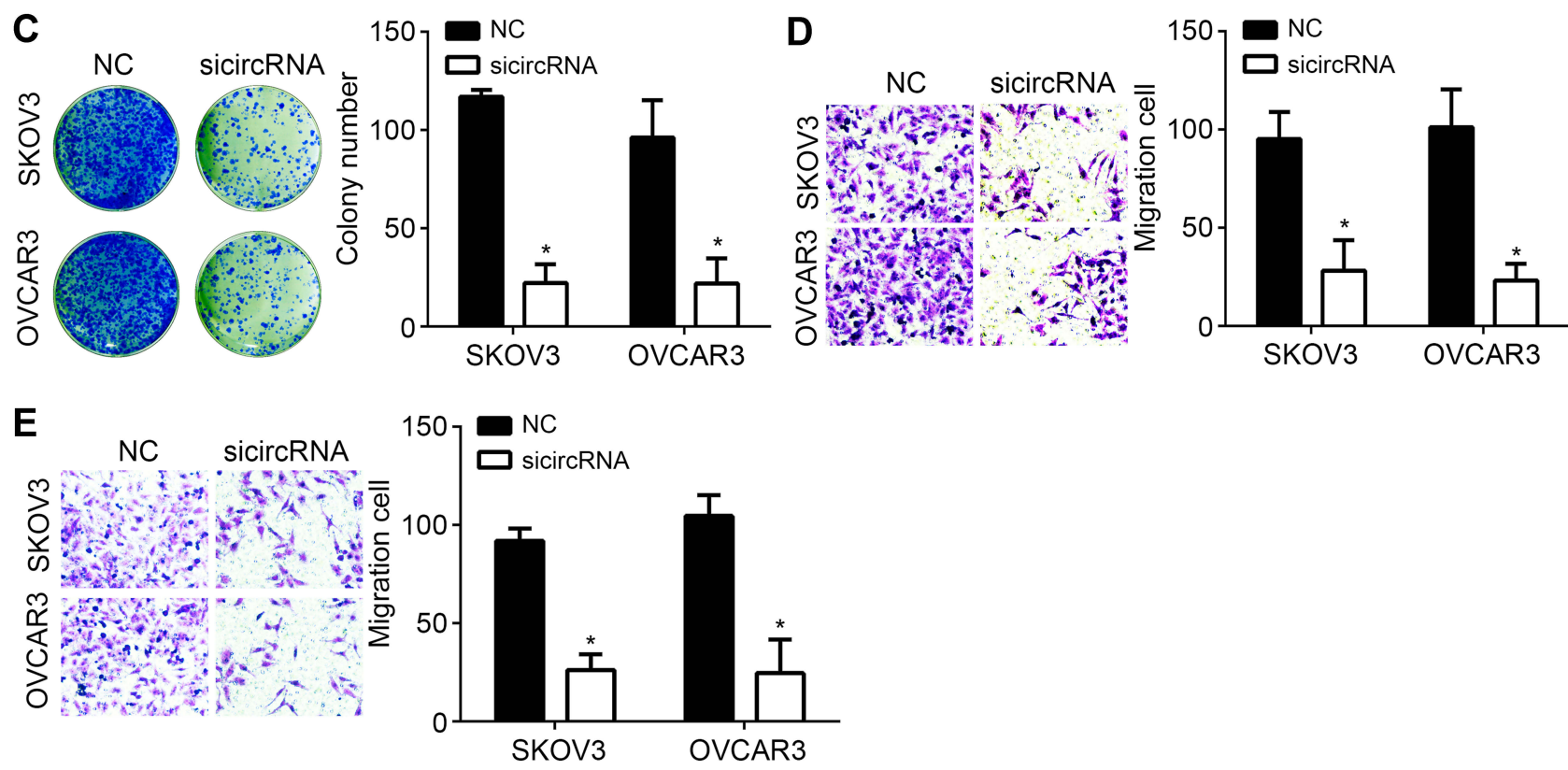

Figure 2 hsa_circRNA_102958 downregulation inhibited OC proliferation, migration and invasion. (A) siRNA transfection reduced hsa_circRNA_I02958 expression in SKOV3 and OVCAR3 cells. (B and C) Cellular proliferation was determined by CCK8 and colony formation assays. (D and E) Migration and invasion were suppressed by hsa_circRNA_102958 downregulation in SKOV3 and OVCAR3 cells. $* \mathrm{P}<0.05$.

\section{hsa_circRNA_I02958 Overexpression Promoted OC Progression}

To further validate the oncogenic roles of hsa_circ RNA_102958, we overexpressed it in SKOV3 and OVCAR3 cells (Figure 3A). We performed functional assays again. CCK8 and colony formation assays also showed that hsa_circRNA_102958 upregulation promoted the proliferation of SKOV3 and OVCAR3 cells (Figure 3B and C). Transwell assay indicated that migration and invasion of SKOV3 and OVCAR3 cells were enhanced by hsa_circRNA_102958 overexpression (Figure 3D and E). Thus, hsa_circRNA_102958 was an important oncogene in OC.

\section{hsa_circRNA_102958 Was a ceRNA for} miR-I 205 to Facilitate SH2D3A Expression We have found that hsa_circRNA_102958 was located in the cytoplasm, implying that hsa_circRNA_102958 may be a ceRNA for miRNAs. We used the bioinformatics analysis through CircInteractome. The ten most potential candidates were identified. Then, we performed luciferase reporter assay using hsa_circRNA_102958 luciferase reporter. We found that only miR-1205 inhibited the activity of hsa_circRNA_102958 reporter (Figure 4A), suggesting miR-1205 may be targeted by hsa_circRNA_102958. To further validate it, we constructed mutant hsa_circRNA_102958 reporter (Figure 4B). Similarly, hsa_circRNA_102958-WT reporter activity was decreased by miR-1205 mimics (Figure 4C). However, miR-1205 had no any effect on hsa_circRNA_102958-Mut reporter (Figure 4C). Additionally, hsa_circRNA_102958 knockdown promoted the expression of miR-1205 and vice versa (Figure 4D and E). And hsa_circRNA_102958 expression was negatively correlated with miR-1205 in OC tissues (Figure 4F). Thus, hsa_circRNA_102958 was the sponge for miR-1205. 
A

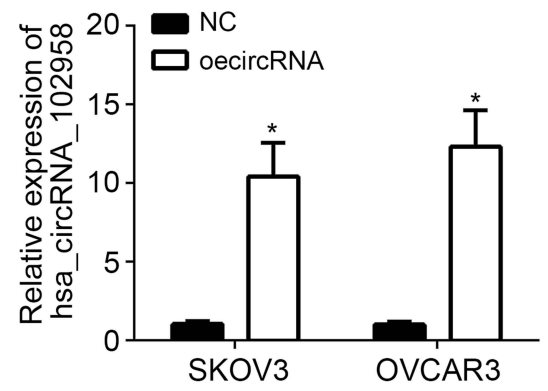

B

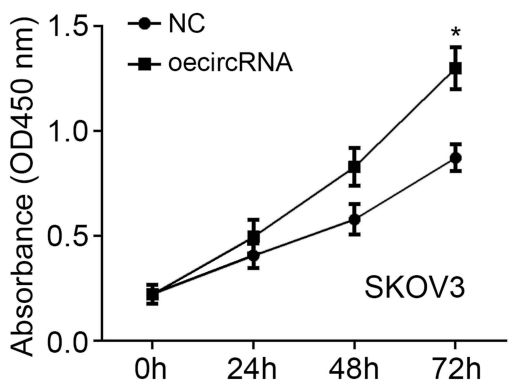

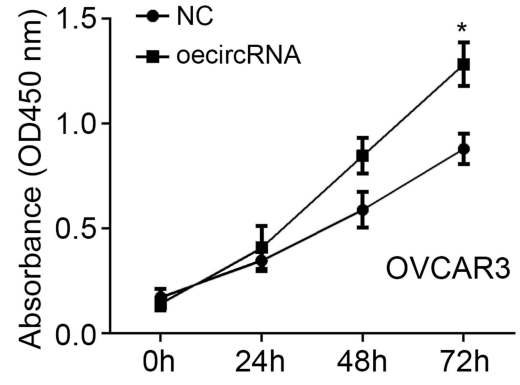

D
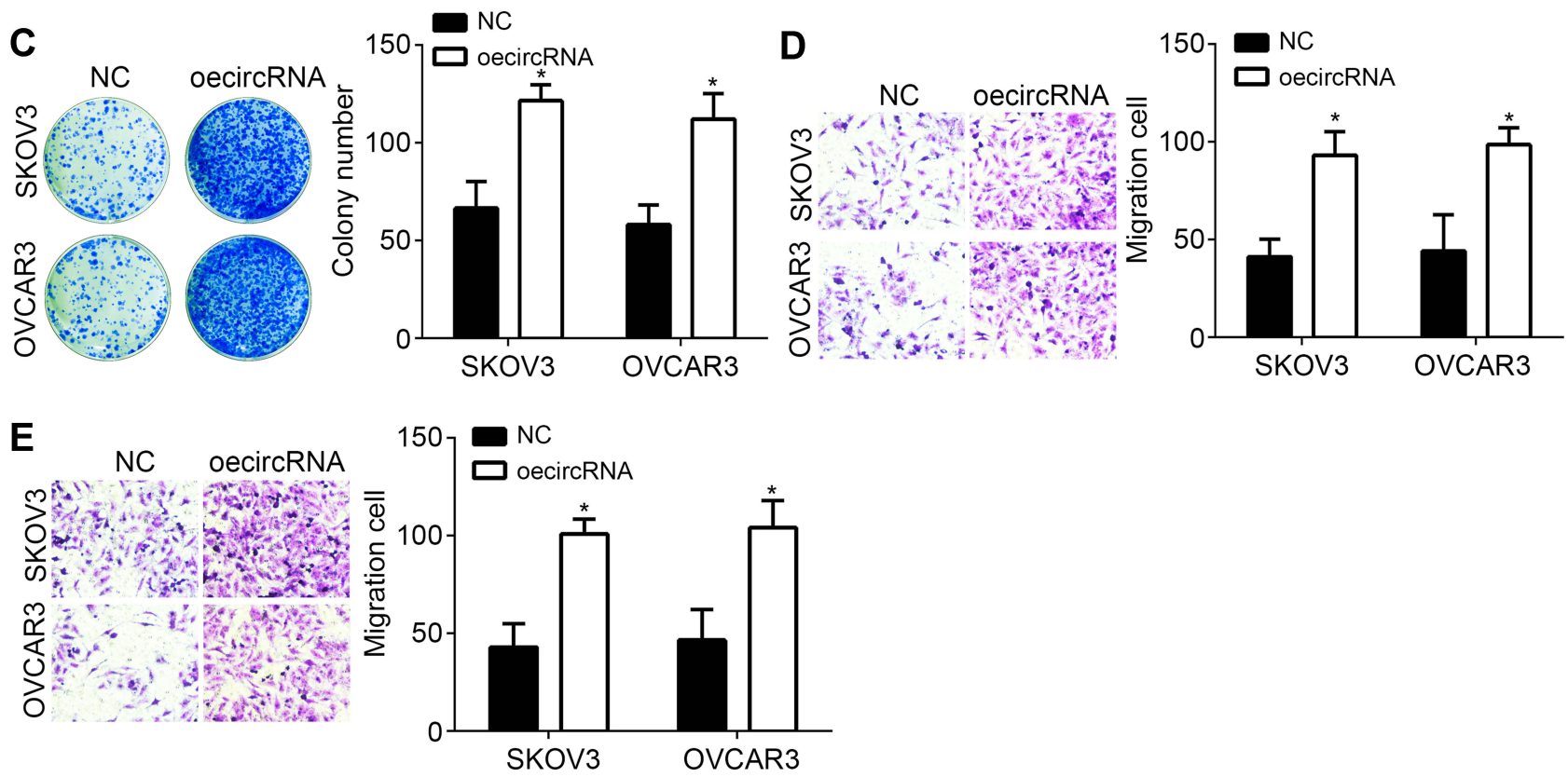

Figure 3 hsa_circRNA_102958 overexpression promoted OC progression. (A) Overexpression efficiency of hsa_circRNA_102958 was confirmed by qRT-PCR. (B and C) hsa_circRNA_102958 overexpression promoted proliferation and colony formation. (D and E) hsa_circRNA_102958 upregulation led to more migration and invasion. $* \mathrm{P}<0.05$.

We further predicted the targets of miR-1205 using bioinformatics analysis (Figure 4G). miRDB and TargetScan tools suggested that SH2D3A was the most potential candidate (Figure 4G). Thus, we constructed WT and Mut SH2D3A luciferase reporter vectors (Figure $4 \mathrm{H}$ ). Luciferase reporter assay demonstrated the interaction between SH2D3A and miR-1205 in OC cells (Figure 4I). Moreover, SH2D3A expression was suppressed by miR-1205 (Figure 4J). Notably, hsa_circRNA_102958 knockdown suppressed the expression of SH2D3A and vice versa (Figure $4 \mathrm{~K}$ and L), which was depending on miR-1205. Moreover, SH2D3A protein level was inhibited by hsa_circRNA_102958 knockdown or miR1205 mimics whereas hsa_circRNA_102958 overexpression or miR-1205 inhibitors promoted SH2D3A expression (Figure 4M and N). Taken together, hsa_circRNA_102958 decoys miR-1205 to promote SH2D3A expression in OC.

\section{hsa_circRNA_I02958/miR-I205/SH2D3A Axis Modulated OC Progression}

Finally, we found that SH2D3A expression was upregulated in OC tissues (Figure 5A and B), indicating an oncogenic role. To further investigate the roles of hsa_circRNA_102958/miR1205/SH2D3A axis, rescue assays were conducted by CCK8 and Transwell assays. Results indicated that miR-1205 inhibitor reversed the inhibitory effects of hsa_circRNA_102958 knockdown on proliferation, migration and invasion (Figure 5C-E). However, SH2D3A knockdown further reversed above trend in tumor cells transfected with sicircRNA and miR-1205 inhibitors (Figure 5C-E). Moreover, SH2D3A overexpression promoted the proliferation, migration and invasion of OC cells and vice versa (Figure 5C-E). In conclusion, hsa_circRNA_102958 promotes OC progression via regulating miR-1205/SH2D3A axis. 


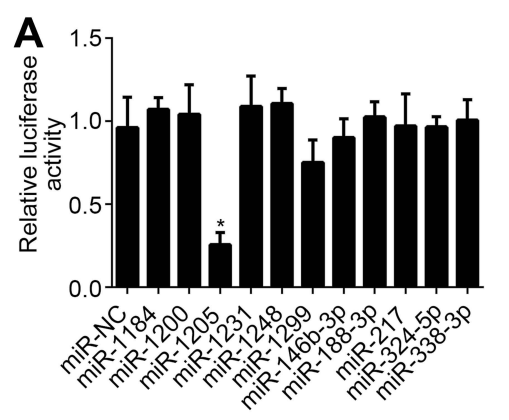

\section{B}
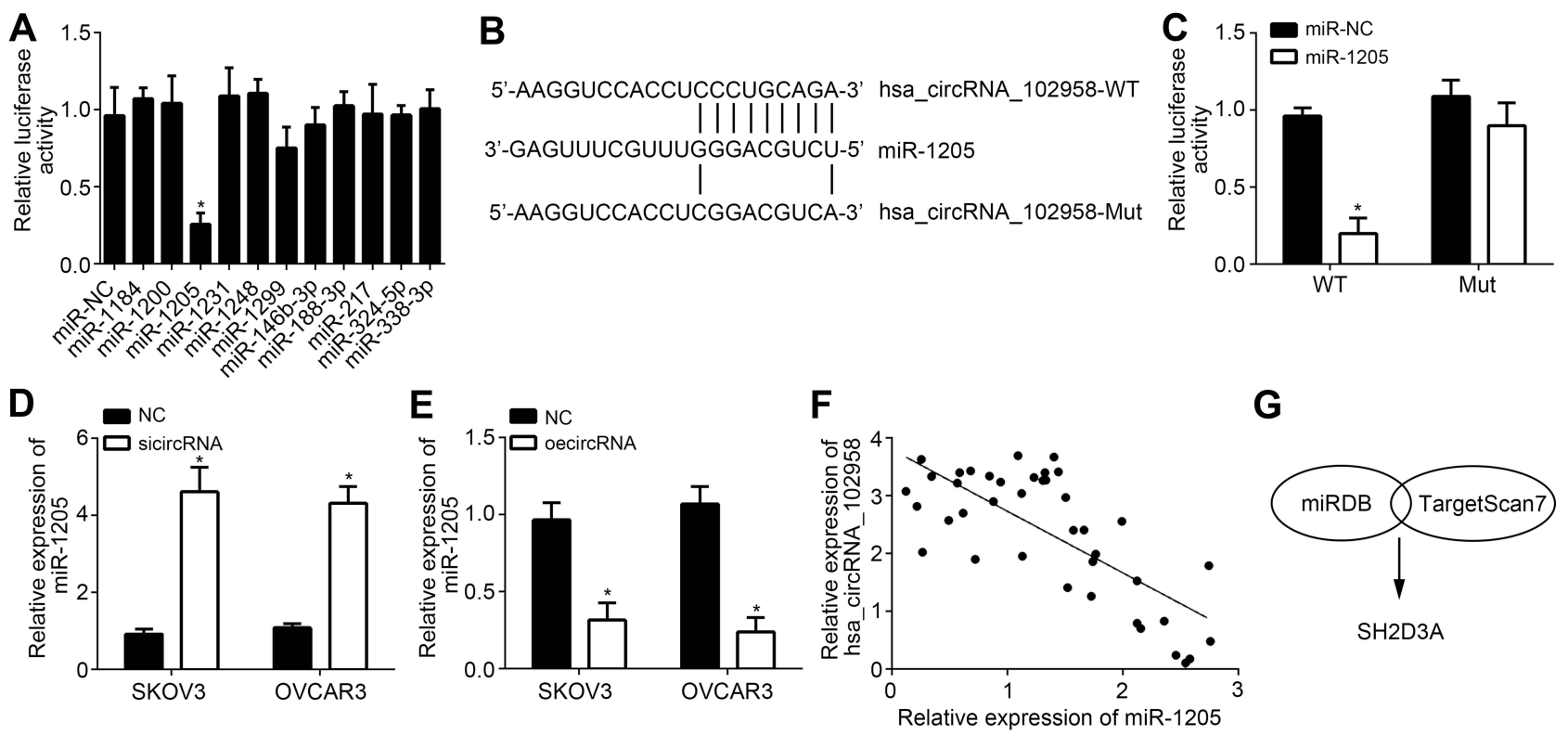

G

H

5'-GUGAACAUGUGACCUGCAGA-3' SH2D3A 3' UTR WT

||||||||

3'-GAGUUUCGUUUGGGACGUCU-5' miR-1205

|

5'-GUGAACAUGUGACGACGUCA-3' SH2D3A 3' UTR Mut
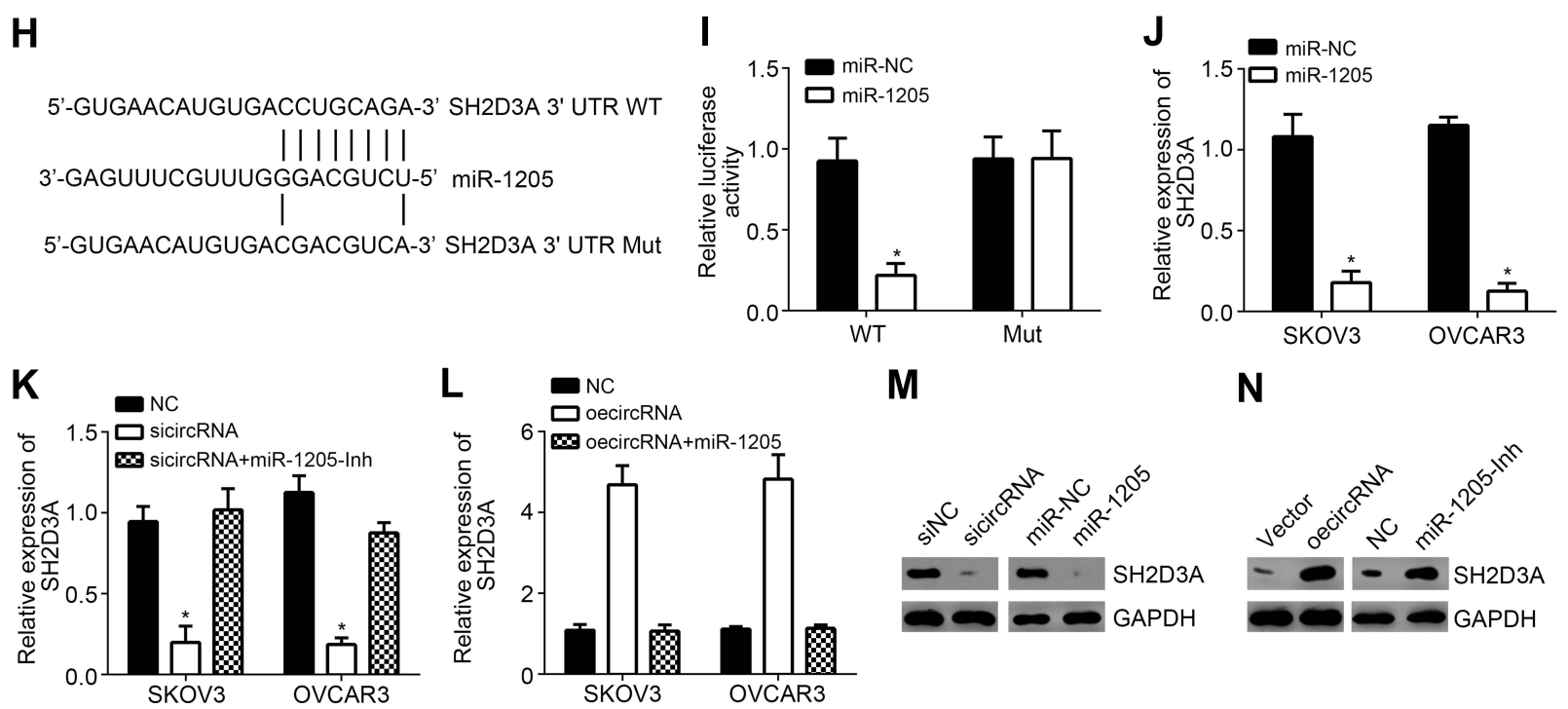

M

$\mathbf{N}$
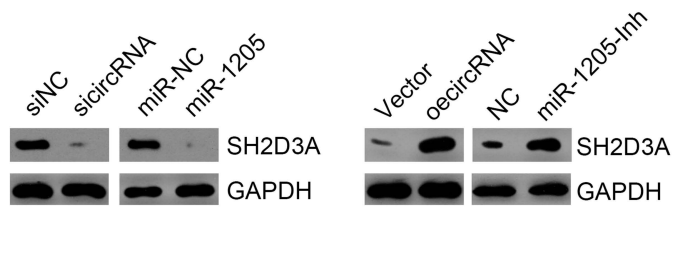

Figure 4 hsa_circRNA_102958 was a ceRNA for miR-1205 to facilitate SH2D3A expression. (A) Luciferase reporter assay implied that miR-1205 mimics suppressed the activity of hsa circRNA 102958 reporter vector in SKOV3 cells. (B) Diagram of the putative binding site with miR-1205 in hsa circRNA 102958. (C) Luciferase reporter assay confirmed the interaction between hsa_circRNA_102958 and miR-1205 in SKOV3 cells. (D and E) hsa_circRNA_102958 knockdown promoted miR-1205 expression and vice versa. (F) Expression correlation between hsa circRNA_102958 and miR-1205 was analyzed in OC tissues. (G) Strategy for prediction of miR-1205 target gene. (H) Diagram of the putative binding site with miR-I 205 in SH2D3A 3'-UTR region. (I) Luciferase reporter assay showed that miR-I 205 inhibited the activity of SH2D3A-WT reporter. (J) miR-1205 inhibited the expression of SH2D3A. (K and L) Expression of SH2D3A was analyzed after transfection with aforementioned plasmids. (M and $\mathbf{N}$ ) Protein level of SH2D3A was measured after transfection with indicated plasmids in SKOV3 cells. ${ }^{*} \mathrm{P}<0.05$.

\section{Discussion}

$\mathrm{OC}$ is an aggressive cancer and accounts for a large portion of cancer-related deaths. ${ }^{12}$ However, its pathogenesis still remains poorly understood. In this study, we found that hsa_circRNA_102958 was highly expressed in OC tissues and cell lines. hsa_circRNA_102958 overexpression was associated with a low survival rate in OC patients. Functionally, hsa_circRNA_102958 knockdown inhibited the proliferation, migration and invasion of $\mathrm{OC}$ cells and vice versa. Increasing studies reveal circRNAs are fine ceRNAs for miRNAs. ${ }^{13}$ We also identified that hsa_circRNA_102958 sponged miR-1205 to promote SH2D3A expression. Our data demonstrated the pivot roles of hsa_circRNA_102958/miR-1205/SH2D3A axis in $\mathrm{OC}$ progression.

Emerging studies have indicated that circRNAs are important regulators in the initiation and progression of human cancers, including OC. ${ }^{14,15}$ For instance, circRNA 
A

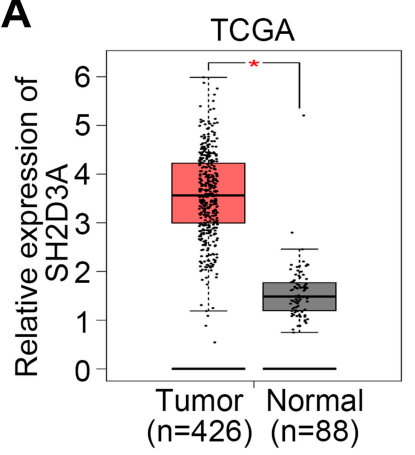

D

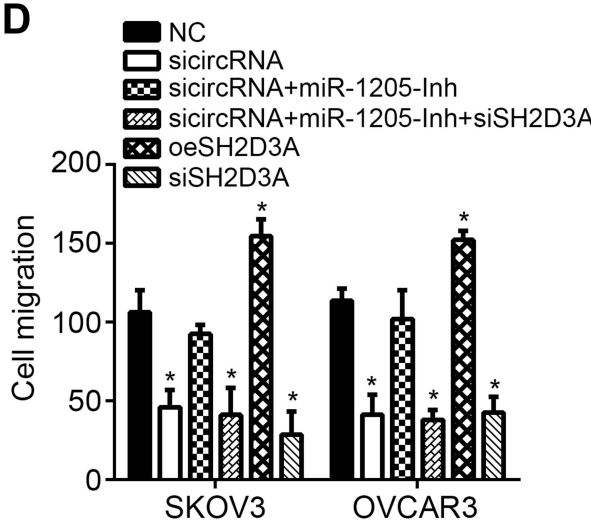

B

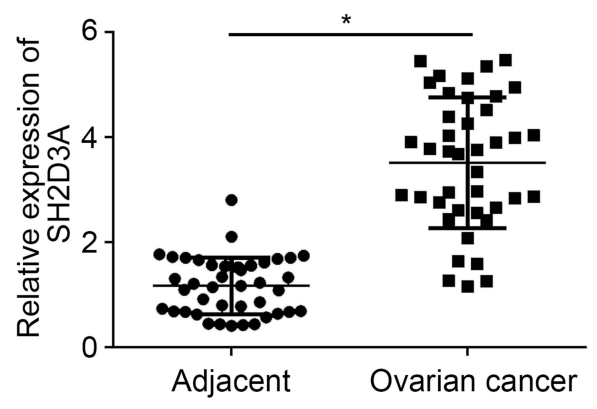

E
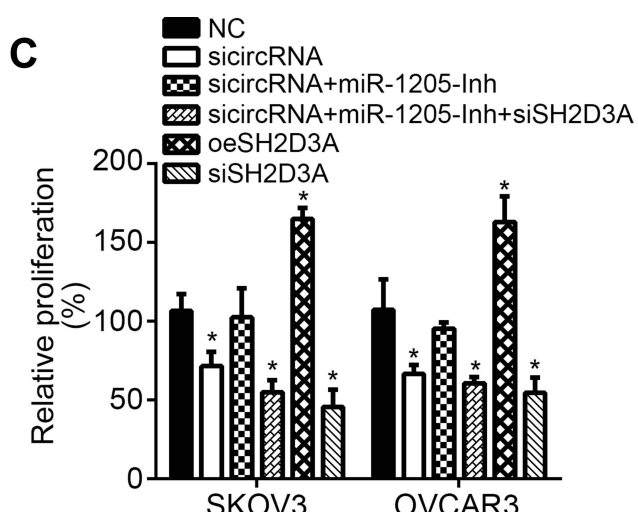

Figure 5 hsa_circRNA_102958/miR-1205/SH2D3A axis modulated OC progression. (A) Relative expression of SH2D3A in OC tissues based on TCGA database. (B) SH2D3A expression was upregulated in OC tissues by qRT-PCR. (C) CCK8 assay was performed to test proliferation after transfection with aforementioned plasmids. (D and E) Transwell assay was conducted to determine migration and invasion after transfection with aforementioned plasmids. $* P<0.05$.

circ-ITCH inhibits OC cell proliferation and invasion via regulating miR-145/RASA1 axis. ${ }^{16}$ hsa_circ_0061140 promotes OC development and metastasis via sponging miR$370 .{ }^{17}$ In addition, circEXOC6B and circN4BP2L2 are identified as potential indicators for prognosis in OC patients. ${ }^{18}$ However, functions of most circRNAs are still uninvestigated. hsa_circRNA_102958 is reported to regulate gastric cancer and colorectal cancer. ${ }^{10,11}$ To our knowledge, its role in other cancers remains unclear. In this study, we identified that hsa_circRNA_102958 was upregulated in OC tissues and cell lines. Moreover, we demonstrated that hsa_circRNA_102958 promoted OC proliferation, migration and invasion. Thus, hsa_circRNA_102958 is a novel oncogenic circRNA in OC.

As well-acknowledged ceRNAs, accumulating researches have demonstrated the interaction between circRNAs and miRNAs. ${ }^{19}$ In our study, we also found that hsa_circRNA_102958 was a sponge for miR-1205. We demonstrated their interaction via luciferase reporter assay. Moreover, we found that hsa_circRNA_102958 knockdown promoted miR-1205 expression and vice versa. Then roles of miR-1205 have been reported in several cancers. A report shows that miR-1205 overexpression promotes osteosarcoma progression. $^{20}$ And miR-1205 upregulation enhances cell growth in lung adenocarcinoma and induces castrationresistance of prostate cancer cells. ${ }^{21,22}$ On the contrary, several evidences indicate that miR-1205 was a tumor suppressor in glioma. ${ }^{23,24}$ Thus, whether miR-1205 acts as an oncogene or tumor suppressor in OC requires to be determined. In our study, we showed that miR-1205 inhibitors promoted the proliferation, migration and invasion of OC cells. Therefore, miR1205 is a new tumor suppressor in OC.

Afterwards, the downstream target of miR-1205 was analyzed by bioinformatics method. We identified SH2D3A and confirmed the interaction between miR-1205 and SH2D3A via luciferase reporter assay. Moreover, we showed that hsa_circRNA_102958 promoted SH2D3A expression via directly inhibiting miR-1205 in OC cells. SH2D3A, also named NSP1, is functionally unclear protein. It has been reported to regulate the c-Jun N-terminal kinase/stressactivated protein kinase pathway. ${ }^{25}$ Nevertheless, whether it regulates tumorigenesis remains unknown. In our study, we found that SH2D3A was upregulated in OC tissues. Moreover, we found that SH2D3A overexpression promoted 
proliferation, migration and invasion of OC cells, indicating its oncogenic roles. Importantly, we performed rescues assays by using siRNAs targeting SH2D3A. We demonstrated that hsa_circRNA_102958 promoted OC progression through miR-1205/SH2D3A axis.

In conclusion, we identified the interactions among hsa_circRNA_102958, miR-1205 and SH2D3A. We demonstrated that hsa_circRNA_102958 facilitated OC cell proliferation, migration and invasion via modulating miR-1205/SH2D3A pathway.

\section{Acknowledgment}

Guan Wang and Huijing Zhang are co-first authors for this study.

\section{Disclosure}

The authors report no conflicts of interest in this work.

\section{References}

1. Jessmon P, Boulanger T, Zhou W, Patwardhan P. Epidemiology and treatment patterns of epithelial ovarian cancer. Expert Rev Anticancer Ther. 2017;17(5):427-437. doi:10.1080/14737140.2017.1299575

2. Cho SY, Kim K, Park MS, et al. Expression of yes-associated protein 1 and its clinical significance in ovarian serous cystadenocarcinoma. Oncol Rep. 2017;37(5):2620-2632. doi:10.3892/or.2017.5517

3. Murali R, Grisham RN, Soslow RA. The roles of pathology in targeted therapy of women with gynecologic cancers. Gynecol Oncol. 2018;148(1):213-221. doi:10.1016/j.ygyno.2017.11.020

4. Moufarrij S, Dandapani M, Arthofer E, et al. Epigenetic therapy for ovarian cancer: promise and progress. Clin Epigenetics. 2019;11 (1):7. doi:10.1186/s13148-018-0602-0

5. Shao F, Huang M, Meng F, Huang Q. Circular RNA signature predicts gemcitabine resistance of pancreatic ductal adenocarcinoma. Front Pharmacol. 2018;9:584. doi:10.3389/ fphar.2018.00584

6. Wu J, Jiang Z, Chen $\mathrm{C}$, et al. CircIRAK3 sponges miR-3607 to facilitate breast cancer metastasis. Cancer Lett. 2018;430:179-192. doi:10.1016/j.canlet.2018.05.033

7. Bai N, Peng E, Xia F, Wang D, Li X, Li X. CircABCC2 regulates hepatocellular cancer progression by decoying MiR-665. J Cancer. 2019;10(17):3893-3898. doi:10.7150/jca.31362

8. Lu Y, Deng X, Xiao G, Zheng X, Ma L, Huang W. circ_0001730 promotes proliferation and invasion via the miR-326/Wnt7B axis in glioma cells. Epigenomics. 2019;11(11):1335-1352. doi:10.2217/epi2019-0121

9. Cai J, Chen Z, Wang J, et al. circHECTD1 facilitates glutaminolysis to promote gastric cancer progression by targeting miR-1256 and activating $\beta$-catenin/c-Myc signaling. Cell Death Dis. 2019;10 (8):576. doi:10.1038/s41419-019-1814-8

10. Wei J, Wei W, Xu H, et al. Circular RNA hsa_circRNA_102958 may serve as a diagnostic marker for gastric cancer. Cancer Biomark. $2019 ; 1-7$.
11. Li R, Wu B, Xia J, Ye L, Yang X. Circular RNA hsa_circRNA_102958 promotes tumorigenesis of colorectal cancer via miR-585/CDC25B axis. Cancer Manag Res. 2019;11:6887-6893. doi:10.2147/CMAR.S212180

12. Lengyel E. Ovarian cancer development and metastasis. Am J Pathol. 2010;177(3):1053-1064. doi:10.2353/ajpath.2010.100105

13. Ouyang H, Chen X, Li W, Li Z, Nie Q, Zhang X. Circular RNA circSVIL promotes myoblast proliferation and differentiation by sponging miR-203 in chicken. Front Genet. 2018;9:172. doi:10.3389/fgene.2018.00172

14. Yang J, Cong X, Ren M, et al. Circular RNA hsa_circRNA_0007334 is predicted to promote MMP7 and COL1A1 expression by functioning as a miRNA sponge in pancreatic ductal adenocarcinoma. J Oncol. 2019;2019:7630894. doi:10.1155/2019/7630894

15. Karedath T, Ahmed I, Al Ameri W, et al. Silencing of ANKRD12 circRNA induces molecular and functional changes associated with invasive phenotypes. BMC Cancer. 2019;19(1):565. doi:10.1186/ s12885-019-5723-0

16. Hu J, Wang L, Chen J, et al. The circular RNA circ-ITCH suppresses ovarian carcinoma progression through targeting miR-145/RASA1 signaling. Biochem Biophys Res Commun. 2018;505(1):222-228. doi:10.1016/j.bbrc.2018.09.060

17. Chen Q, Zhang J, He Y, Wang Y. hsa_circ_0061140 knockdown reverses FOXM1-mediated cell growth and metastasis in ovarian cancer through miR-370 sponge activity. Mol Ther Nucleic Acids. 2018;13:55-63. doi:10.1016/j.omtn.2018.08.010

18. Ning L, Long B, Zhang W, et al. Circular RNA profiling reveals circEXOC6B and circN4BP2L2 as novel prognostic biomarkers in epithelial ovarian cancer. Int $J$ Oncol. 2018;53(6):2637-2646. doi:10.3892/ijo.2018.4566

19. Guan YJ, Ma JY, Song W. Identification of circRNA-miRNA-mRNA regulatory network in gastric cancer by analysis of microarray data. Cancer Cell Int. 2019;19(1):183. doi:10.1186/s12935-019-0905-Z

20. Wu Z, Shi W, Jiang C. Overexpressing circular RNA hsa_circ_0002052 impairs osteosarcoma progression via inhibiting Wnt/ $\beta$-catenin pathway by regulating miR-1205/APC2 axis. Biochem Biophys Res Commun. 2018;502(4):465-471. doi:10.1016/j. bbrc.2018.05.184

21. Dai B, Kong DL, Tian J, Liu TW, Zhou H, Wang ZF. microRNA1205 promotes cell growth by targeting APC2 in lung adenocarcinoma. Eur Rev Med Pharmacol Sci. 2019;23 (3):1125-1133. doi:10.26355/eurrev_201902_17003

22. Wang Y, Li X, Liu W, et al. MicroRNA-1205, encoded on chromosome 8q24, targets EGLN3 to induce cell growth and contributes to risk of castration-resistant prostate cancer. Oncogene. 2019;38 (24):4820-4834. doi:10.1038/s41388-019-0760-3

23. Yang M, Li G, Fan L, Zhang G, Xu J, Zhang J. Circular RNA circ_0034642 elevates BATF3 expression and promotes cell proliferation and invasion through miR-1205 in glioma. Biochem Biophys Res Commun. 2019;508(3):980-985. doi:10.1016/j.bbrc.2018.12.052

24. Yi C, Li H, Li D, et al. Upregulation of circular RNA circ_0034642 indicates unfavorable prognosis in glioma and facilitates cell proliferation and invasion via the miR-1205/BATF3 axis. J Cell Biochem. 2019;120(8):13737-13744. doi:10.1002/jcb.28646

25. Lu Y, Brush J, Stewart TA. NSP1 defines a novel family of adaptor proteins linking integrin and tyrosine kinase receptors to the c-Jun $\mathrm{N}$-terminal kinase/stress-activated protein kinase signaling pathway. J Biol Chem. 1999;274(15):10047-10052. doi:10.1074/jbc.274. 15.10047 


\section{Publish your work in this journal}

Cancer Management and Research is an international, peer-reviewed open access journal focusing on cancer research and the optimal use of preventative and integrated treatment interventions to achieve improved outcomes, enhanced survival and quality of life for the cancer patient.
The manuscript management system is completely online and includes a very quick and fair peer-review system, which is all easy to use. Visit http://www.dovepress.com/testimonials.php to read real quotes from published authors.

Submit your manuscript here: https://www.dovepress.com/cancer-management-and-research-journal 